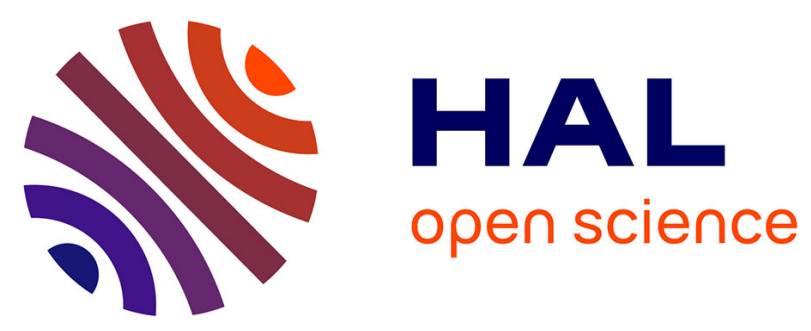

\title{
Anisotropic build-up of photo-induced waveguides in biased photorefractive media
}

\author{
Virginie Coda, Charles Ciret, T. Mengis, M. Gorram, Germano
}

Montemezzani, Mathieu Chauvet, Fabrice Devaux

\section{To cite this version:}

Virginie Coda, Charles Ciret, T. Mengis, M. Gorram, Germano Montemezzani, et al.. Anisotropic build-up of photo-induced waveguides in biased photorefractive media. Optics Communications, 2015, 355, pp.382-390. 10.1016/j.optcom.2015.07.005 . hal-01244346

\section{HAL Id: hal-01244346 \\ https://hal.science/hal-01244346}

Submitted on 13 May 2020

HAL is a multi-disciplinary open access archive for the deposit and dissemination of scientific research documents, whether they are published or not. The documents may come from teaching and research institutions in France or abroad, or from public or private research centers.
L'archive ouverte pluridisciplinaire HAL, est destinée au dépôt et à la diffusion de documents scientifiques de niveau recherche, publiés ou non, émanant des établissements d'enseignement et de recherche français ou étrangers, des laboratoires publics ou privés. 


\title{
Anisotropic build-up of photo-induced waveguides in biased photorefractive media
}

\author{
Virginie Coda ${ }^{\mathrm{a}, \mathrm{b}}$, Charles Ciret $^{\mathrm{a}, \mathrm{b}, 1}$, Thibault Mengis ${ }^{\mathrm{b}, \mathrm{a}}$, Mohamed Gorram $^{\mathrm{a}, \mathrm{b}}$, Germano Montemezzani ${ }^{\mathrm{a}, \mathrm{b}}$, Mathieu Chauvet ${ }^{\mathrm{c}}$, \\ Fabrice Devaux ${ }^{\mathrm{c}}$ \\ ${ }^{a}$ Université de Lorraine, LMOPS, EA 4423, 2 rue E. Belin, Metz, F-57070, France \\ ${ }^{b}$ CentraleSupélec, LMOPS, EA 4423, 2 rue E. Belin, Metz, F-57070, France \\ ${ }^{c}$ FEMTO-ST Institute, UMR CNRS 6174, University of Franche-Comté, $15 B$ Avenue des Montboucons, F-25000 Besanon, France
}

\begin{abstract}
We study the anisotropic dynamic formation of photo-induced waveguide in biased photorefractive media by means of a time resolved three-dimensional numerical model of the space-charge field formation. Specific features of the dynamic creation of 1D-confined planar and 2D-confined channel waveguides are elucidated and confirmed experimentally. Thanks to a transient space-charge field overshooting in both $1 \mathrm{D}$ and $2 \mathrm{D}$ geometries, light confinement in the first dimension occurs at a very early stage in the formation of the space-charge field. In the 2D channel type waveguides, the final shape of the waveguide index contrasts and modes depend not only on the illumination geometry and intensity distribution, but also on the anisotropy of the charge mobility along the two transverse directions.
\end{abstract}

Keywords: Photorefractive material, photo-induced structures, waveguides

\section{Introduction}

Reconfigurable or semi-permanent optical waveguides can be realized by means of the photoinduced refractive index changes arising from the photorefractive (PR) effect either in a longitudinal [1, 2, 3], or a lateral illumination configuration of an appropriate biased crystal $[4,5,6,7]$. The former technique is based on the self-focusing mechanism of the recording light and leads to waveguides, which are essentially straight or only slightly bended. In contrast, for the lateral illumination technique the shape of the waveguides are defined by an external mask or spatial light modulator, that permits to design specific structures allowing for instance to test analogies between quantum physics and optics [8], or to realize broadband integrated multiple beam splitters [9]. With lateral illumination, 1D planar type waveguide can be realized by using a single illumination light stripe [4], while 2D-confined channel-type waveguides require a more complex control illumination of the crystal sample from two perpendicular directions, one of which through transparent electrodes $[7,10]$. It was observed that the dynamics of formation of such 2D waveguides exhibit a strong anisotropic character. The guided light confines first along the direction of the applied electric field and reaches a nearly round shape only at a much later stage [7]. A qualitative explanation of this behavior was given in [7] on the base of a highly simplified argumentation that assumed a purely one dimensional charge transport and neglected the effects of charge diffusion in the photorefractive material. Nevertheless, a deeper understanding of the observed dynamics can only be obtained by a more complete

\footnotetext{
Email address: coda50univ-lorraine.fr (Virginie Coda)

${ }^{1}$ Present adress: OPERA-Photonique, Université libre de Bruxelles (ULB), 50 av. F. D. Roosevelt, CP 194/5, B-1050 Bruxelles, Belgium
}

model that takes into account charge transport in all possible directions as well as the anisotropic properties of the material.

Indeed the creation of a space-charge electric field and a refractive index modulation by the PR process is a highly complex phenomenon that depends on several anisotropic tensorial properties of the involved material, such as the dielectric, the mobility, or the electro-optic tensor, just to name a few [11]. In addition, the experimental use of a bias electric field (or the presence of an intrinsic photogalvanic effect) leads to an additional symmetry breaking that favors the charge transport in that particular direction with respect to the others. All this leads to a complex build-up dynamics of the space-charge distribution $[12,13,14,15]$, which, will depend also on the spatial geometry of the writing beam.

In this paper we aim at understanding the anisotropic dynamics of formation of photoinduced $2 \mathrm{D}$ channel waveguides recorded by lateral illumination with the procedure described in [7]. For this purpose we use a full 3D numerical model that was successfully applied to describe complex dynamics of photorefractive self-focusing beams in different materials $[16,17,18]$. Section 2 deals with the theoretical background and the numerical model used to simulate the PR waveguide formation. The results of the transient simulations comparing the various cases (1D-, 2D- confinement, lateral vs. longitudinal illumination) are presented in section 3. Finally, section 4 discusses the influence of various parameters on the shape and guiding properties of photo-induced channel waveguides obtained by a crossed lateral illumination. An anisotropy of the conductivity as well as the intensities of the control light beams are shown to influence these properties both in the transient dynamic regime and at steady-state. It is shown that the experimentally observed anisotropy of the formation dynam- 
ics is enhanced by an initial overshooting of the space-charge field on both sides of the waveguide in the direction of the externally applied electric field (direction $x$ ). This overshooting leads to a transient dip of the refractive index on both sides of the waveguide core and contributes to accelerate the guided wave confinement in this direction with respect to the perpendicular $y$-direction. On the other hand, charge-diffusion along the transverse $y$-axis partially washes out the index variation in this direction, specially if the transverse charge mobility $\mu_{y}$ is large. All these features cannot be extracted from the simple argumentation presented earlier in [7].

\section{Theoretical background and numerical model}

We base our description of charge transport and creation of a space-charge electric field by the PR effect on the standard Kukhtarev-Vinetskii model [19, 20], which assumes the presence of a single impurity level in the band gap of the material as well as a single charge carrier. We neglect the influence of photogalvanic currents, which is justified in the case of the $\mathrm{Sr}_{0.61} \mathrm{Ba}_{0.39} \mathrm{Nb}_{2} \mathrm{O}_{6}$ (SBN) used in our experiments. The crystal is assumed to possess a total deep donor density $N_{D}$, of which $N_{D}^{+}$are initially ionized. In the virgin state, charge neutrality is insured by an equivalent number of acceptors $N_{A}$, with $N_{D}^{+}=N_{A}$ at the initial time $t=0$. We also assume that the density of conducting electrons $N_{e}$ is small compared to $N_{A}$ at all times, and that the creation time of conducting electrons is much faster than the characteristic evolution time of the space charges in the deep level. Both these assumptions are very well satisfied for our experimental conditions in SBN.

Under the above assumptions, following the treatment given in [16], the Kukhtarev-Vinetskii equations can be brought in the normalized form

$$
\begin{gathered}
\tilde{N}_{e}=\frac{\xi\left(I+I_{d}\right)\left(\tilde{N}_{D}-\tilde{N}_{D}^{+}\right)}{\tilde{N}_{D}^{+}}, \\
\tilde{N}_{D}^{+}=1+\tilde{\rho}, \\
\frac{\partial \tilde{\rho}}{\partial t}=-\nabla \cdot\left(\tilde{N}_{e}[\mu] \mathbf{E}\right)-\frac{k_{B} T}{e} \nabla \cdot\left([\mu] \nabla \tilde{N}_{e}\right) .
\end{gathered}
$$

Here $\tilde{N}_{e}<<1$ is the free electron density normalized to the acceptor density $N_{A}$, and $\tilde{N}_{D}$ and $\tilde{N}_{D}^{+}$are the total and empty donor densities normalized in the same way. The quantity $\tilde{\rho}$ is the space charge density normalized to $e N_{A}$ (with $e$ the elementary charge of an electron). The driving quantity in the above equations is the light intensity $I=I(x, y, z)$ which gives the light intensity distribution associated to the control beams that initiate the charge redistribution process. The quantity $I_{d}$ is the equivalent dark intensity giving free electrons in absence of the control beams, due to thermal excitation and/or the presence of a homogeneous background illumination. The constant $\xi$ is defined as $\xi \equiv s / \gamma N_{A}$, where $s$ is the photo-excitation coefficient that describes the probability of photo-excitation from the donors to the conduction band, and $\gamma$ is the recombination constant proportional to the probability of recombination from the conduction band to the deep level. Note that the anisotropy of the electron mobility is taken explicitly into account by using a tensorial mobility $[\mu]$. Finally, $k_{B}$ and $T$ are the Boltzmann constant and the absolute temperature, respectively.

In order to obtain the evolution of the space charge field $\mathbf{E}$ for a given light distribution, the above set of equations is iteratively solved starting from initial conditions. To begin with, $\tilde{N}_{e}$ is calculated from Eq. (1) assuming $\tilde{N}_{D}^{+}(0)=1$. From $\mathrm{Eq}$.(3) the space charge variation is then calculated at a time step $\Delta t$. The corresponding ionized donor density is then calculated from Eq. (2).

The next step is the calculation of the space charge field distribution E. This is done by using the classical electrostatic equation for the electric displacement field $\mathbf{D}$ created at a point $\mathbf{r}=(x, y, z)$ of the crystal by the charge distribution $\rho\left(\mathbf{r}^{\prime}\right) d^{3} \mathbf{r}^{\prime}$ in the medium volume $\mathrm{V}$,

$$
\mathbf{D}(\mathbf{r})=\epsilon_{0}[\epsilon] \mathbf{E}(\mathbf{r})=\frac{1}{4 \pi} \iiint_{V} \rho\left(\mathbf{r}^{\prime}\right) \frac{\mathbf{r}-\mathbf{r}^{\prime}}{\left|\mathbf{r}-\mathbf{r}^{\prime}\right|^{3}} d^{3} \mathbf{r}^{\prime},
$$

where $\epsilon_{0}$ is the vacuum permittivity and $[\epsilon]$ is the material static dielectric tensor. This approach has the advantage of an easier numerical implementation in an anisotropic medium than the Poisson equation frequently used in PR models. Equation. (4) is solved by numerical integration over the crystal volume thanks to 3D numerical Fourier transforms (FFT). Specifically, the convolution of Eq. (4) is found by taking an FFT of both constituting functions, multiplying pointwise, and then performing an inverse FFT, which finally permit to determine the three components of the electric field vector $\mathbf{E}=\left(E_{x}, E_{y}, E_{z}\right)$ at the time $t$.

Finally the space-charge field $\mathbf{E}$ induces a change in the index ellipsoid through the Pockels effect given by

$$
\Delta\left(\frac{1}{n^{2}}\right)_{i j}=\sum_{k} r_{i j k} E_{k}
$$

where $E_{k}$ are the electric field components and $r_{i j k}$ the linear electro-optic coefficients of the considered material. The modified refractive index distribution is finally used to determine the spatial evolution of the light beam guided in the waveguide structure being formed. This is done by means of a Beam Propagation Method algorithm (FFT-BPM) that simulates the propagation of a probe beam in the crystal under the PR perturbation.

The whole procedure is repeated at each temporal steps $\Delta t$ until the final time. A normalized time $\tau$ is defined by $\tau=$ $t / T_{d}$ where $T_{d}$ is the ( $x$-oriented) dielectric response time of the material under illumination [21] given as

$$
T_{d}=\frac{\epsilon_{0} \epsilon_{x x}}{\sigma_{x}^{0}}=\frac{\epsilon_{0} \epsilon_{x x}}{e \mu_{x} N_{e}^{0}},
$$

with $\epsilon_{x x}$ the permittivity of the material along $x$ (which is the principal charge drift direction), $\sigma_{x}^{0}$ the electric conductivity in this direction given by $e \mu_{x} N_{e}^{0}$, with $\mu_{x}$ the corresponding mobility, and $N_{e}^{0}$, the electron density. The latter is calculated from Eq. (1) with $I=I_{0}$, where $I_{0}$ is the light intensity of the control beam in the center of the illumination. 


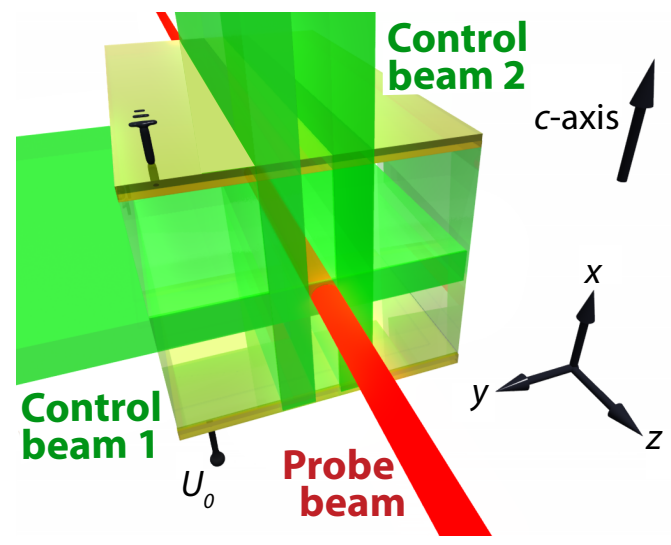

Figure 1: Illumination of the PR crystal for recording and testing lightinduced waveguides by lateral illumination according to the procedure developed in $[7,10]$. For the realization of a planar waveguide only the control beam 1 is switched on. Both control beams are required to realize a channel waveguide. Note that the control beam 2 is composed of two light stripes. The electric field is applied along the crystal $c$-axis through semi-transparent electrodes.

\section{Anisotropic dynamics of waveguide formation: $1 D$ vs 2D}

The model presented in the previous section is now applied to predict the anisotropic build-up dynamics of the PR spacecharge field under different illumination configurations. For our numerical simulations we consider parameters for a biased $\mathrm{Sr}_{0.61} \mathrm{Ba}_{0.39} \mathrm{Nb}_{2} \mathrm{O}_{6}$ (SBN:61) crystal illuminated by spatially structured 532-nm light beam(s) to create the refractive index distribution. For the simulations we use the parameters $s=4 \times 10^{-6} \mathrm{~m} \mathrm{~s}^{-1} \mathrm{~W}^{-1}, \gamma=10^{-6} \mathrm{~m}^{3} \mathrm{~s}^{-1}$ [22] and for the acceptor and donor concentrations we choose $N_{A}=2 \times 10^{22} \mathrm{~m}^{-3}$ and $N_{D} / N_{A}=50$. The anisotropy of electron mobility is considered with $\mu_{x}=2.7 \times 10^{-6} \mathrm{~m}^{2} \mathrm{~V}^{-1} \mathrm{~s}^{-1}$ and $\mu_{y}=\mu_{z}=0.42 \mu_{x}$ [23]. The dark intensity $I_{d}$ is fixed at $3 \mathrm{~mW} \mathrm{~cm}^{-2}$. This high value models the experimentally used incoherent background illumination that illuminates the crystal to limit the saturation of the PR index change.

The effect of the photo-induced index change on the propagation of a light beam is simulated by a low intensity cw laser beam at $633 \mathrm{~nm}$ which doesn't induce any PR effect when traveling in the $z$-direction. Its linear polarization is oriented along the crystal $c$-axis (direction $x$ ), parallel to the direction of the applied electric field $E_{0}$ set to $3 \mathrm{kV} \mathrm{cm}^{-1}$. The latter is required in order to obtain an optimum waveguide with a local maxima of the refractive index in the waveguide region. For this configuration the largest electro-optic coefficient $\left(r_{33}\right)$ coefficient of SBN:61 plays the major role for the index perturbation, but smaller coefficients $r_{13}$ and $r_{42}$ are also considered since we take into account the vectorial orientation of the space-charge field $\mathbf{E}$ in the three dimensions.

A schematic 3D representation of the system under investigation is shown on Fig. 1, where the control beams are used to generate the waveguide which is tested by the probe beam. Planar and channel waveguides will be analyzed. For a 1D waveguide only the control beam 1 is switched on, both control beams coming through perpendicular crystal surfaces are required to realize a $2 \mathrm{D}$ waveguide. The case of a circular longitudinal control beam, corresponding to a beam inducing a PR effect superposed to the probe beam, will be also considered (but not shown on Fig. 1). All control beams are invariant upon translation in z-direction for the realization of straight waveguides. Therefore, the parameters and variables associated to the PR effect, such as the control beam intensity $I$, the space-charge field $\mathbf{E}$ or the refractive index change $\Delta n$ will have no dependence on $z$. Only the probe beam amplitude distribution will explicitly depend on the $z$-coordinate.

\subsection{Planar waveguide}

We first consider the realization of a planar-type waveguide obtained by the simultaneous application of the electric field and of the control beam composed of a light stripe parallel to the $y z$-plane (control beam 1 on Fig. 1). The shape of the control beam is chosen as a super gaussian, $\left.I(x)=I_{0} \exp \left(-\left(x / w_{0}\right)^{4}\right)\right)$ with $w_{0}=20 \mu \mathrm{m}$ and $I_{0}=5 \mathrm{~mW} \mathrm{~cm}{ }^{-2}$, in order to approximate the experimentally realized shape. The calculated build-up of the space-charge and of the related electric field distribution is illustrated in Fig. 2, where $\tilde{\rho}, E_{x}, E_{y}$ and $I$ are depicted in the transverse $x y$-plane. The control beam profile is shown on Fig. 2(f). The normalized charge distribution $\tilde{\rho}$ is represented at two characteristic times: $t=0.2 T_{d}$ (early stage of the process) and $t=3 T_{d}$ (close to the stationary state) in Fig. 2(a) and in Fig. 2(c), respectively. As expected, the resulting charge distribution $\tilde{\rho}$ is anti-symmetric relative to the center of the control beam, as a result of the drift-dominated (electron) charge transport. The strongest component $E_{x}$ of the space-charge field is the one parallel to the externally applied field and is shown in Fig. 2(b) for $\tau=0.2$ and in Fig. 2(d) for $\tau=3$. For completeness, at this final time the transverse $E_{y}$ component is also shown (Fig. 2(e)), however, in the present 1D configuration this component vanishes everywhere. As expected by the 1D analytical model of the PR effect, at steady state (Fig. 2(d)) a minimum of $E_{x}$ is found in the center of the control illumination, where the light intensity is maximal, due to the screening of the applied field $E_{0}$. The choice of a ratio $I_{0} / I_{d}$ close to one leads nevertheless to a partial screening of the applied electric field. In contrast the component $E_{x}$ stays close to $E_{0}$ far from the illuminated region because the distance between the electrodes is much larger than the extension of the control beam in $x$-direction.

A peculiar and less expected behavior is observed in the transient regime at the beginning of the process. Indeed a local overshooting of the space charge field occurs at both edges of the control beam, where the component $E_{x}$ becomes significantly stronger than $E_{0}$ (red part in Fig. 2(b)). Even though a $1 \mathrm{D}$ situation is considered, these local maxima of $E_{x}$ remind the characteristic profile along the polar axis expected at steadystate in the case of a 2D-type illumination [24]. However, in the presently discussed 1D configuration the transient maxima disappear in the steady state. This is seen in Fig. 2(d), where the profile of $E_{x}(x)$ corresponds to the one expected by steady-state analytical models, for which the space-charge field is expected to follow $E_{x}(x)=E_{0} I_{d} /\left(I_{d}+I(x)\right)$. This means that $E_{x}(x)$ finally reaches a profile that essentially mimics the lateral illumination $I(x)$. The time-resolved simulations of the 1D illumination of 

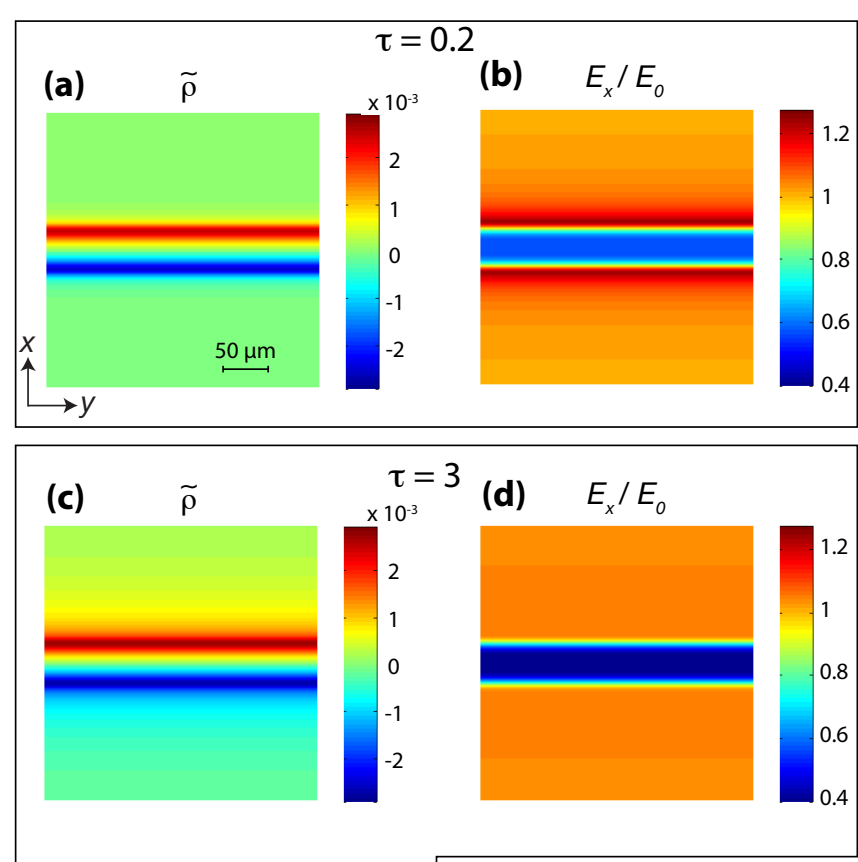

(e) $E_{y} / E_{0}$

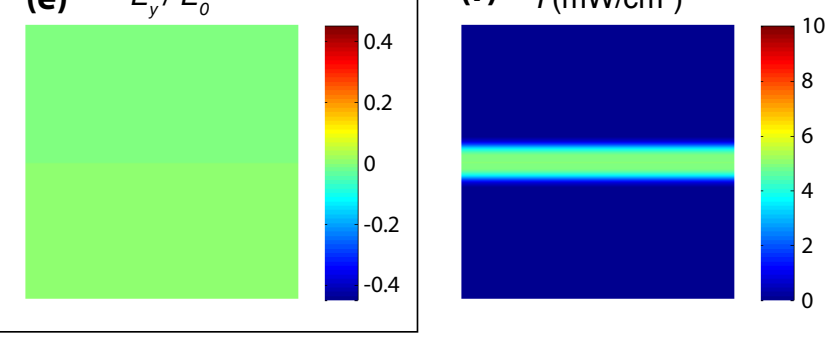

Figure 2: Build-up of a planar type PR waveguide under 1D stripe illumination and a bias field of $E_{0}=3 \mathrm{kV} \mathrm{cm}$. Two particular times are considered: the beginning of the process at $\tau=0.2(\mathrm{a}, \mathrm{b})$ and the quasi-steady-state regime at $\tau=3$ (c,d,e). For these two instants, the normalized charge density distribution $\tilde{\rho}$ is reported on (a) and (c), and the main component of the space charge field $E_{x}$ in (b) and (d). The second, transverse component $E_{y}$ of the space-charge field is given at the final time in (e). The configuration of the control beam $I$ is shown in (f). All diagrams are invariant with respect to $z$.

a PR crystal indicates therefore a richer dynamic than expected by commonly used 1D stationary type models.

An alternative representation of the dynamic PR-waveguide formation can be gained by plotting the evolution of the $x$ - and $y$-profiles of the PR-field-induced refractive index change $\Delta n$ (extracted from Eq. (5)). For a probe wave polarized along $x$, that gives the largest index contrast, and a 1D stripe illumination, these profiles are shown at different chosen times in Fig. 3(a) for the same parameters as in Fig. 2. The progressive screening of the field $E_{0}$ modifies the variation $\Delta n$ at the location of the beam (around $(x, y)=(0,0))$. In the present specific situation the $y$-profile $\Delta n(0, y)$ is always flat and its constant value gets close to zero by approaching the steady-state. Zero is not rigorously reached due to the partial screening of the field because of the high dark intensity. The refractive index tends to go back to the unperturbed one in this region. Indeed, even if all the components of the electro-optic tensor are taken into account in the calculations (with Eq. (5)), the main contribution
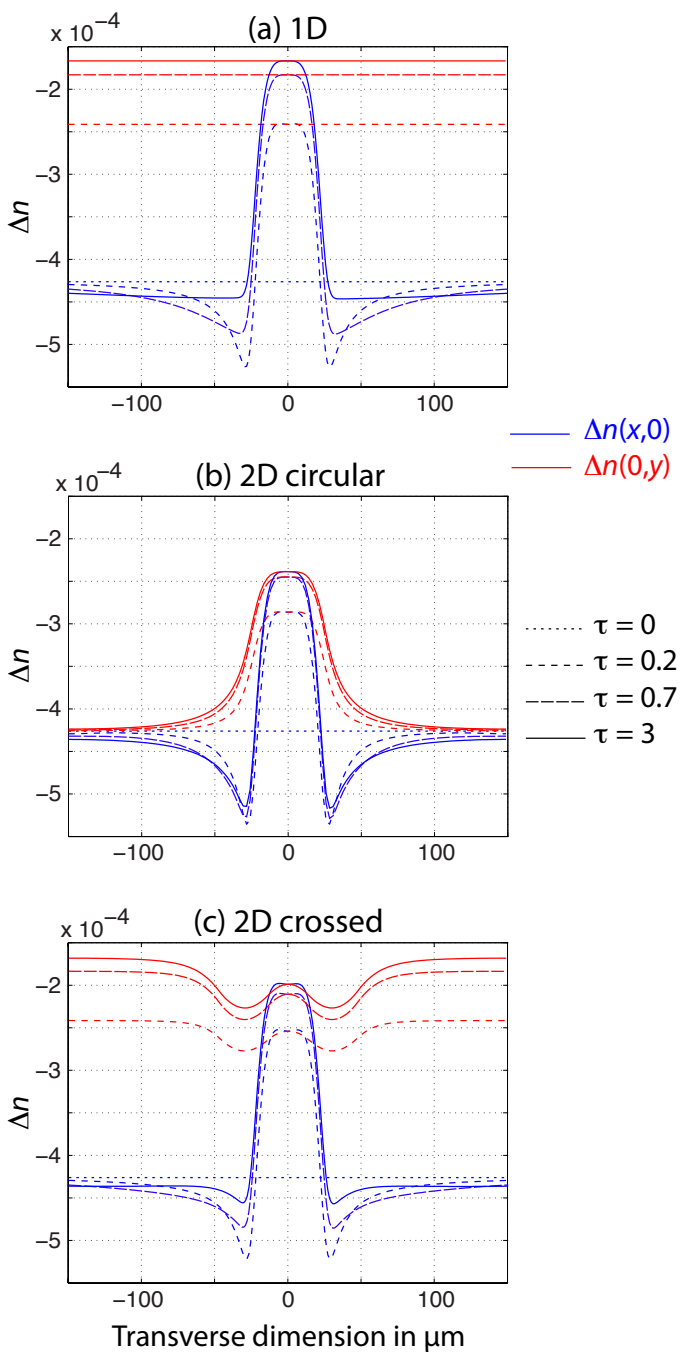

Figure 3: Time evolution of the PR-induced refractive index change profiles $\Delta n$ under different types of illuminations. (a) 1D stripe illumination in the conditions of Fig. 2. (b) Circular 2D illumination as in Fig. 5. (c) 2D side illumination as in Fig. 6. The refractive index change is calculated for a $x$-polarized probe beam. The value $\Delta n=0$ corresponds to the unperturbed refractive index $n \equiv n_{e}$ of the material. The $\Delta n$ profiles are plotted along $x$ for $y=0$ (bottom bue curves) and along $y$ for $x=0$ (top red curves). $(x, y)=(0,0)$ corresponds to the center of the transverse representations of Figs. 2, 5 and 6.

of the electro-optic effect, for the considered polarization, can be approximated by $\Delta n \approx-1 / 2 n_{e}^{3} r_{33} E_{x}$. This show that the applied field $E_{0}$ leads to a reduction of the extraordinary refractive index $n_{e}$ all over the crystal, but in the regions where this field is being screened. The transient overshooting of $E_{x}(x)$ discussed above is translated here in the two dips in the $x$-profile $\Delta n(x, 0)$ seen in Fig. 3(a) for the earlier times. Dynamically these dips get deeper until approximately $t=0.2 T_{d}$. Then they tend to decay before they finally disappear completely in the steady-state. A focus on this behavior is illustrated on Fig. 4 that shows the time evolution of the minimum of $\Delta n(x, 0)$ for the three specific illumination situations considered in this paper. The 1D illumination case (red dashed line in Fig. 4), discussed in this section, shows that the depth of the dip reaches nearly $10^{-4}$ (as referred 


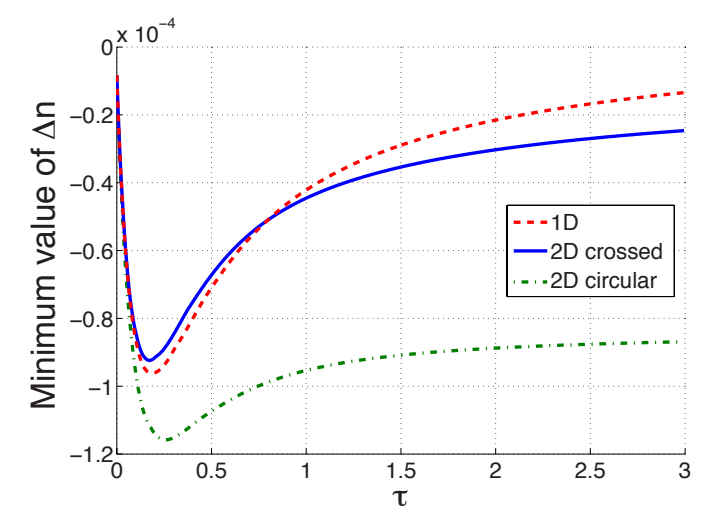

Figure 4: Temporal evolution of the minimum of $\Delta n$ for the three considered types of illuminations used for Fig. 3. The minimum of $\Delta n$ corresponds to the difference between $\Delta n$ in the dark regions far from the beam and the minimum of $\Delta n(x, 0)$ along $x$ for $\mathrm{y}=0$.

to the difference between $\Delta n$ in the dark regions and the minimum of $\Delta n$ ) before approaching again zero at very long times.

The above simulations show that an efficient refractive index contrast is obtained much earlier than the steady-state regime, even if the profile of $\Delta n$ continues to evolve. Indeed even though in the initial part of the build-up the field in the center of the illuminated region is not yet fully screened (refractive index not yet at its maximum), the appearance of the two transient index depressions on the edges of the waveguide allow to reach a sufficient refractive index contrast for a strong confinement of the probe wave already at early times.

\subsection{Longitudinal $2 D$ circular illumination}

For comparison, the PR characteristics obtained created by a circular symmetric 2D illumination are reported on Fig. 5. The same conditions as in Fig. 2 are used. Only the geometry of the illumination $I(x, y)$ is changed to $I(x, y)=$ $I_{0} \exp \left(-\left(\sqrt{x^{2}+y^{2}} / w_{0}\right)^{4}\right)$. This means that, in this case, the control beam is launched in the same longitudinal direction than the probe beam represented on Fig.(1). Figure 5(b) shows that the overshootings of the component $E_{x}$ along the vertical $x$-axis are present also in this case. However, unlike for the case of the 1Dillumination, here they persist also in the final state (Fig. 5(d)). In contrast, $E_{x}$ is always smaller than $E_{0}$ on the horizontal line passing through the center of the beam $(x=0)$, at any time during the dynamics. This character of the distribution for a 2D illumination has long been known [15, 25, 24]. Note that the quadrupole character of the profile of $E_{y}$, seen in Fig. 5(e) is associated with two current loops from each side of the light beam [24]. As mentioned above, here the dips remain present in the steady-state as seen clearly in the bottom blue $x$-profiles $\Delta n(x, 0)$ in (Fig. 3(b)). The green dashed-dotted line in Fig. 4 shows indeed that a minimum of $\Delta n$ is reached at almost the same time than for the case of a 1D illumination, but at longer time it remains almost unchanged. We may define the refractive index contrast useful for waveguide confinement in $x$ direction as $\Delta n_{x} \equiv \Delta n(0,0)-\min [\Delta n(x, 0)]$. Interestingly we find that for identical peak intensities for the $1 \mathrm{D}$ and $2 \mathrm{D}$ configurations,
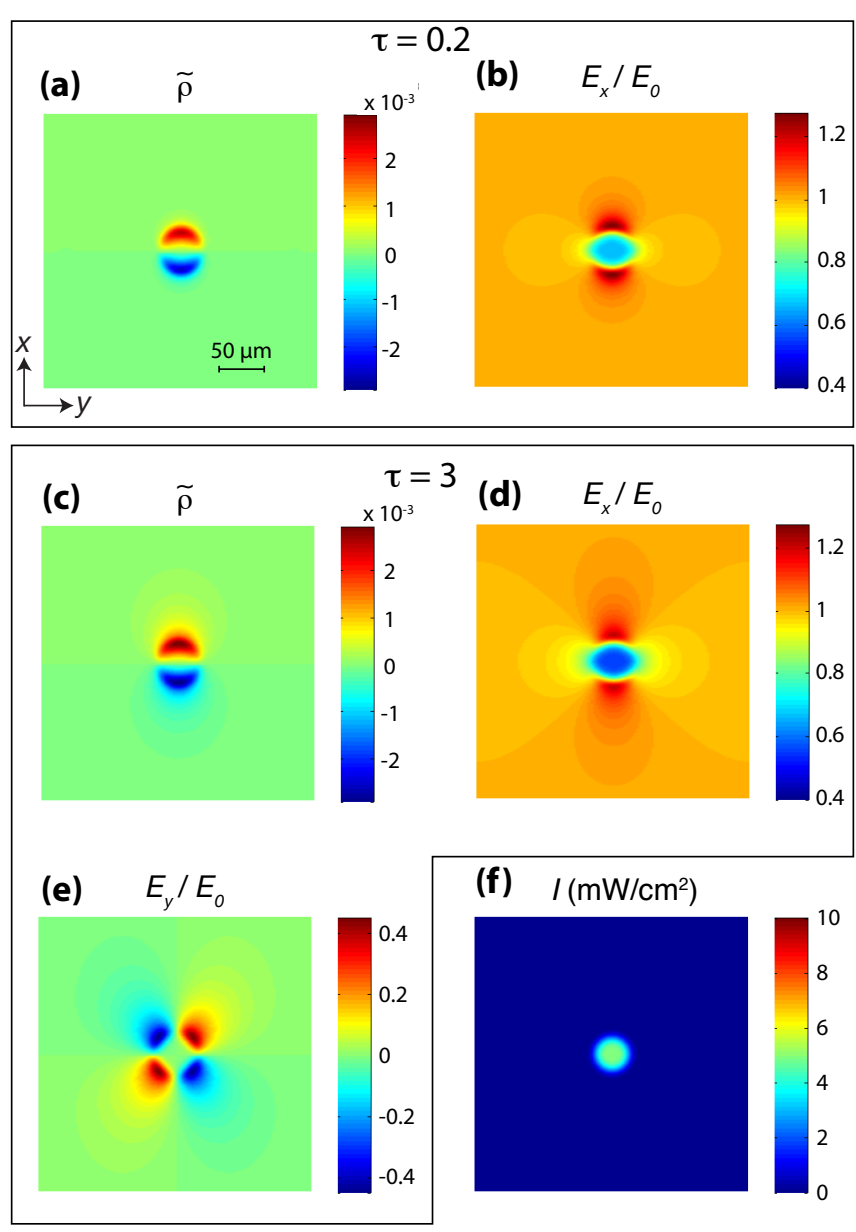

Figure 5: Build-up of space charges and space-charge field in the case of a 2D circular symmetric illumination. The same representations and the same times as in Fig. 2 are given. Except for the illumination represented in (f), all parameters are identical to those in Fig. 2. Note that the color scales for $E_{x}$ and $E_{y}$ are not the same.

this refractive index contrast is nearly the same in both configurations, even though $\Delta n(0,0)$ in the center part of the beam is different: the field screening is less efficient for the $2 \mathrm{D}$ configuration but this is compensated by deeper minima.

The 2D light distribution that we have discussed in this section merits some comments. Indeed this 2D illumination induces a refractive index change suitable for the realization of a channel waveguide, but this distribution cannot be obtained by a lateral illumination of the PR crystal. The above circular intensity distribution can be obtained only by a longitudinal illumination of the crystal along $z$ by using a focused beam. Experimentally, illumination patterns being invariant in the $z$ direction are easily produced by lateral illumination but cannot be so easily obtained by a longitudinal illumination, where diffraction of the focused beam will occurs during the propagation over centimeter-long-crystals. Propagating non-diffracting beams can be realized if the conditions are set to realize a PR spatial solitons, but this will add supplementary constrains to the shape of the waveguide. On the one hand, 2D waveguides induced by lateral illumination require a more sophisticated 
structuring of the illumination than required by self-focused beam techniques. On the other hand, lateral illumination gives more freedom and versatility to the 3D structured geometry of the waveguide. In the next section our discussion will concentrate on channel waveguides formed by such a $2 \mathrm{D}$ lateral illumination method [7].

\subsection{Channel waveguide with lateral illuminations}

The realization of a channel waveguide by side illuminations is obtained by a second control beam entering the crystal perpendicularly to the first one (see Fig. 1) [7] and composed of two light stripes parallel to the $x z$-plane. Since the $x$-direction is parallel to the crystal $c$-axis and to the applied electric field, transparent electrodes are required. The role of the orthogonal control beam 2 is very different to the one of control beam 1. Photo-induced free charges generated by the beam 2, getting through the entire crystal, will essentially create a kind of optically induced pseudo-short circuit between the electrodes and no refractive index change is therefore obtained by a single stripe of light in this plane. Nevertheless when crossing two light stripes parallel to the $x z$-plane with one parallel to the $y z$-plane as illustrated in Fig. 1, in the central part (where the probe beam propagates) one gets a local minimum of the space charge field (corresponding to a local maximum of the refractive index). This is due to the fact that the transverse beam 2 can be seen as an additional local dark intensity for the beam 1. As a consequence the field screening is less effective where both control beams are present. This leads to a lower refractive index where both beam intersect than where only control beam 1 is present. Therefore in the central part, at $(x, y, z)=(0,0, z)$, a local maximum of refractive index is obtained with respect to both transverse directions, which gives a channel waveguide. This has been explained in more detail in Ref. [7] and will be discussed further here by means of our 3D numerical calculations.

For the modeling of the present configuration we use the light intensity distribution $I(x, y)=I_{1} \exp \left(-\left(x / w_{0}\right)^{4}\right)+I_{2}[\exp (-((y-$ $\left.\left.\left.\left.y_{0}\right) / w_{2}\right)^{4}\right)+\exp \left(-\left(\left(y+y_{0}\right) / w_{2}\right)^{4}\right)\right]$, with $w_{2}=13.5 \mu \mathrm{m}, w_{0}=$ $20 \mu \mathrm{m}$ and $y_{0}=2.25 w_{0}$. The intensity $I_{1}$ is associated to control beam 1 , while $I_{2}$ is associated to control beam 2 . In this section we will limit our discussion to analyze the situation where the two control beam peak intensities are identical $\left(I_{1}=I_{2}=I_{0}=\right.$ $5 \mathrm{~mW} \mathrm{~cm}^{-2}$ ) (Fig. 6(f)). The results are depicted in Fig. 6 for the distributions of $\tilde{\rho}$ and $\mathbf{E}$ and in Fig. 3(c) and Fig. 4 for the index change.

As seen in Fig. 6(a) and Fig. 6(c), the presence of the control beam 2 partially and locally erases the charge distribution accumulated in a 1D geometry due to the light stripe of the control beam 1. In the transient state, the overshooting of $E_{x}$ along $x$ can be observed again (Fig. 6(b)). This overshooting disappears in the steady-state as for the 1D waveguide, except near the light stripe of beam 2, where the illumination is not of $1 \mathrm{D}$ type anymore (Fig. 6(d)). So in these locations, in the steadystate regime, the profile of the space charge field conserves a $2 \mathrm{D}$ character. This is confirmed by the distribution of the $E_{y}$ component (Fig. 6(e)) which is associated again with current loops, and has the form of double quadrupole.
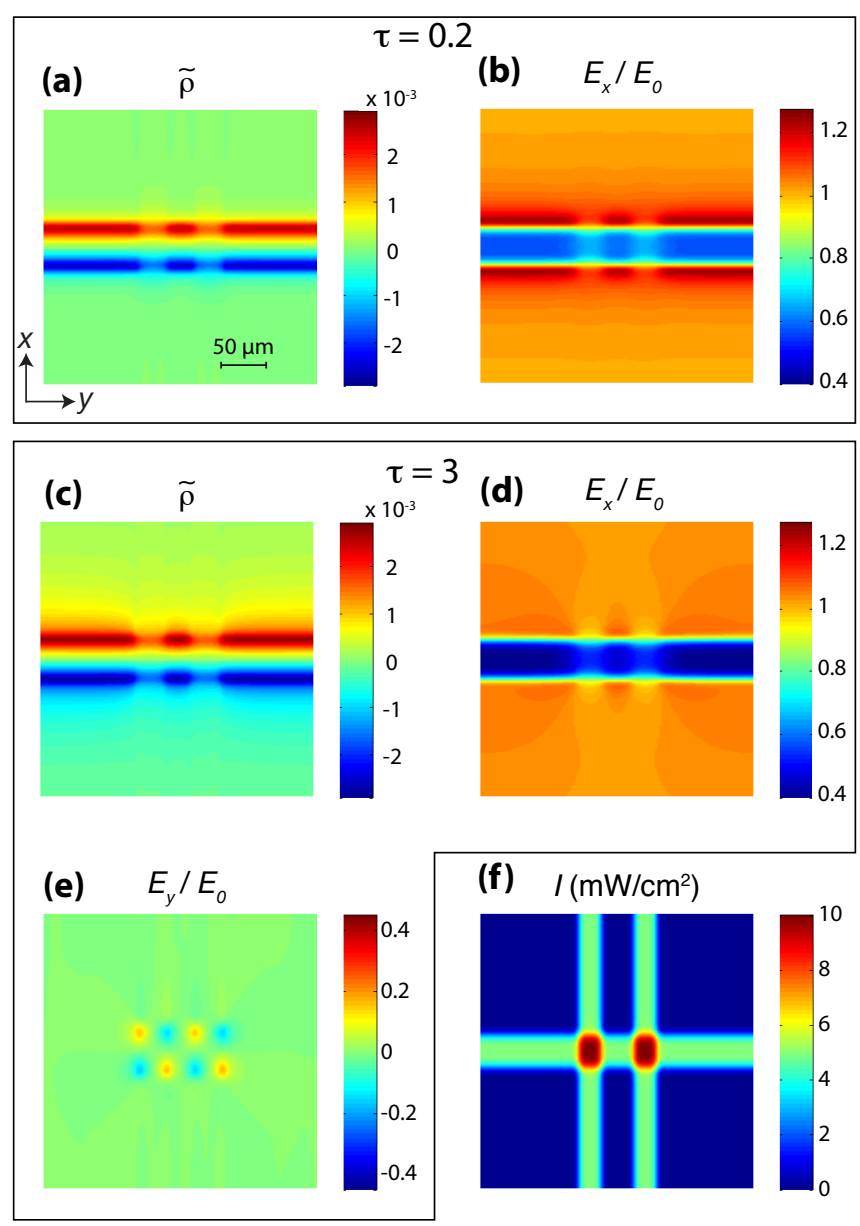

Figure 6: Build-up of space charges and space-charge field in the case of 2D waveguide induced by crossed lateral illumination. The same representations and the same times as in Fig. 2 are given. Except for the illumination represented in (f), all parameters are identical to those in Fig. 2.

The solid line in Fig. 4 shows that the temporal evolution of the depth of the refractive index dips closely resemble the one found for the 1D planar situation, which confirms that in the central part, the temporal dynamics is close to the one for the $1 \mathrm{D}$ case. However, comparison of the solid and the dashed line shows that the relaxation is now partial. This is due to the remaining local 2D character associated with the breaking of the translational symmetry along the $y$ axis. Finally, Fig. 3(c) visualizes how a local maximum of the refractive index is obtained in both the $x$ and $y$ dimensions, thus inducing the wished channel waveguide. The index contrast of the waveguide along $y$ direction, $\Delta n_{y} \equiv \Delta n(0,0)-\min [\Delta n(0, y)]$, is smaller than $\Delta n_{x}$ (defined in the previous section). A highly simplified model such as the one proposed in Ref. [7] predicts that for the same peak intensity $I_{1}=I_{2} \gg I_{d}$ and for rectangular illumination profiles $\Delta n_{y}=0.5 \Delta n_{x}$. The waveguide refractive index contrast ratio $C=\Delta n_{y} / \Delta n_{x}$ obtained here is smaller due to several parameters, taken into account in our more realistic simulations and is analyzed in the following section. 


\section{Characteristics of PR channel waveguides by lateral illu- minations}

\subsection{Influence of dark intensity and tensorial conductivity}

We first note that the above ratio $C$ depends on the value of the "dark intensity" $I_{d}$, which is related either to a thermal dark conductivity or to a homogeneous photoconductivity induced by a background illumination, or by a combination of the two. Using the proper value for $I_{d}$ is important in order to avoid the widening of the induced waveguides into regions where only spurious intensities of the control beams exist, which happens for too small value of $I_{d}$. If its value is too large, it limits the waveguide refractive index contrast both along the $x$ and $y$-directions. And then as shown in our simulations the value of $C$ decreases. This feature was already revealed in the simplified model of Ref. [7] which predicted $\Delta n_{x} \propto 1 / I_{d}$ and $\Delta n_{y} \propto 1 / I_{d}^{2}$ for large $I_{d} \gg I(x, y)$. In the simulations of section 3 a high value of the dark intensity, $I_{d}=3 \mathrm{mWcm}^{-2}$ has been used to fit the experimental conditions. This has the consequence that the obtained ratio between $\Delta n_{y}$ and $\Delta n_{x}$ is roughly $C=0.11$ (see Fig. 3(c)), which is significantly smaller than 0.5 , the value expected from [7] by considering only the ratio of the control beam intensities $I_{1}$ and $I_{2}$ with $I_{d}=0$. For the realistic case simulated in Fig. 3(c) the predicted ratio from [7] is $C=0.38$, the discrepancy with the above value $C=0.11$ is due to both the effect of the dips on the side of the waveguide discussed above and the effect of diffusion in the transverse $y$-direction allowed by a finite mobility $\mu_{y}$ taken into account with $\mu_{y} / \mu_{x}=0.42$.

Indeed one key parameter to the build-up of PR dynamics is obviously the electrical conductivity. We show below that the conductivity not only dictates the speed of the PR buildup dynamics but its anisotropy also influences the ratio $C$. In photorefractive models treating one dimensional situations, for instance when recording a grating by interference of two plane waves, the transverse carrier mobility can be ignored due to symmetry considerations. In contrast, in the 2D situations corresponding to Fig. 5 and Fig. 6 the transverse charge transport by diffusion as well as drift in the field $E_{y}$ cannot be neglected anymore. Therefore the role of the quantity $\mu_{y}$ must be analyzed in more detail. Simulations have been conducted by varying the value of $\mu_{y}$ to observe its influence on the ratio $C$ in the steady state regime $(\tau=10)$. The dark intensity $I_{d}$ has been reduced to $0.1 \mathrm{~mW} \mathrm{~cm}^{-2}$ in order to limit the effect of the background illumination. The results are summarized on Fig. 7. The nearly exponential decrease of $C$ upon increasing ratio $\mu_{y} / \mu_{x}$ confirms how the charge transport along $y$ is detrimental to the index contrast in this direction. A large $\mu_{y}$ leads to a less effective field screening in the center of the guiding region since the refractive index does not fully recover the value in absence of the applied field. This is also connected to a much weaker modulation of the refractive index in the transverse direction (see the inset for $\mu_{y} / \mu_{x}=2$ on Fig. 7). On the contrary, by imposing $\mu_{y}=0$ one finds that $C$ reaches its highest value, and the value of $\Delta n(0,0)$ in the center of the waveguide recovers the value of the unbiased material. For the parameters of Fig. 7 with no transverse mobility $\left(\mu_{y}=0\right)$ one obtains $C=0.34$, which differs from the value $C=0.5$ predicted for $I_{1}=I_{2} \gg I_{d}$ by the simple model of

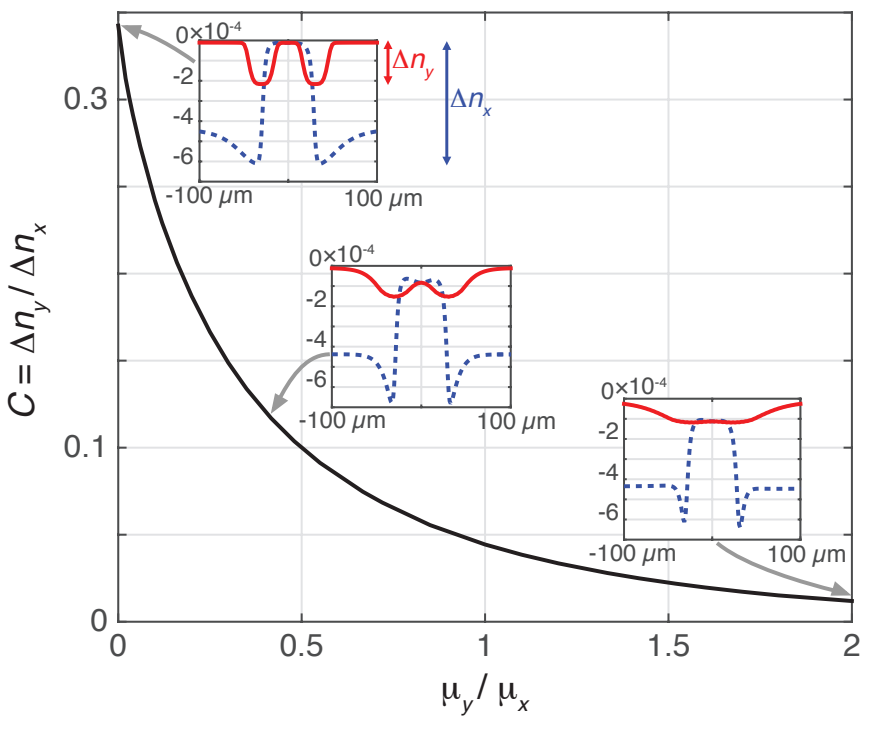

Figure 7: Influence of the transverse mobility $\mu_{y}$ on the waveguide refractive index contrast ratio $C=\Delta n_{y} / \Delta n_{x}$ at $\tau=10$. The control beam illuminations, $E_{0}$ and the crystal parameters are identical to Fig. 6 except $I_{d}=0.1 \mathrm{~mW} \mathrm{~cm}^{-2}$ and $\mu_{y}$ that varies. The refractive index profile corresponding to $\mu_{y} / \mu_{x}=0 ; 0.42$ and 2 are represented in the insets. $\Delta n(0, y)$ is represented with the solid red line and $\Delta n(x, 0)$ is the blue dash profile.

Ref. [7]. Again, the reason is principally due to the remaining dip in the $x$-profile which has the effect of decreasing the value as already mentioned.

\subsection{Theoretical and experimental guiding properties vs. inten- sity ratio}

We now focus our attention on the guiding properties of the photo-induced waveguides generated by crossed lateral illumination discussed above where a small value of $C$ is obtained even at steady-state. A probe beam at the wavelength of $633 \mathrm{~nm}$ is launched in the 2D waveguide. In simulations it is assumed that this beam does not affect the refractive index distribution. Experimentally this hypothesis is insured by using a sufficiently weak cw He-Ne laser beam. The probe beam is focused at the input face of the crystal (Full-Width-at-HalfMaximum, FWHM=17 $\mu \mathrm{m}$ ) at the location where the waveguide is induced. The propagating beam is linearly polarized along $x$ to get the maximum refractive index change. It is injected simultaneously to the application of the control beams and of the applied field $E_{0}$ to monitor the dynamic formation of the waveguide.

The charge distribution, the electric field and the refractive index change calculated in section 3.3 and depicted in Fig. 6 and Fig. 3(c) are used to simulate the evolution of the probe beam by the BPM algorithm. This allows us to predict the beam size at the output after propagation in a $1-\mathrm{cm}$ long crystal. Results are reported on Fig. 8(a) for both transverse dimensions. The plots in Fig. 8(a) show the beam width evolution until $t \approx 0.18 T_{d}$, which corresponds approximately to the duration of the experimental evolution described below. After this time there is almost no further evolution of the output beam profile even though the index distribution has not yet reached 
(a)

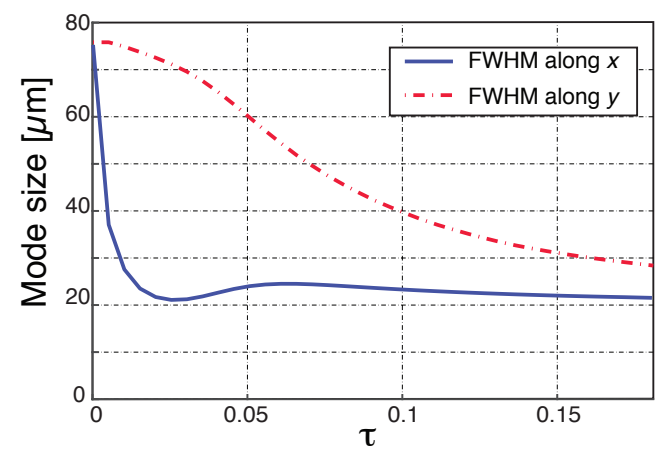

(b)

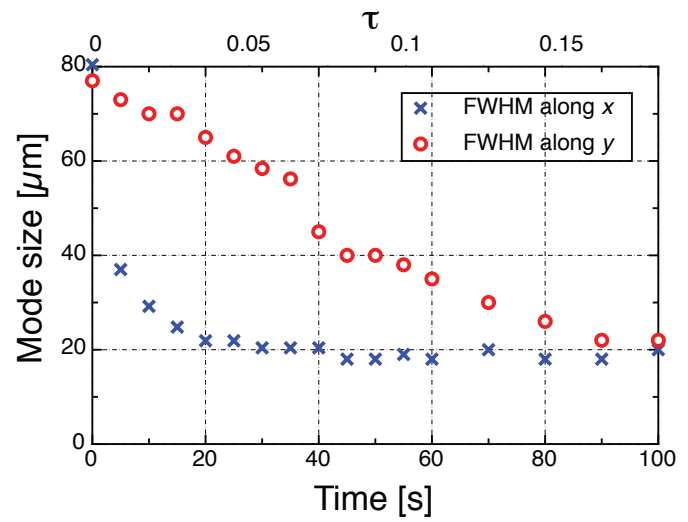

Figure 8: Time evolution of the probe beam size after propagation in the 1-cmlong SBN sample during the formation of a channel waveguide with a lateral illumination of $I_{2}=I_{1}=5 \mathrm{~mW} \mathrm{~cm}^{-2}$ and $E_{0}=3 \mathrm{kV} \mathrm{cm}^{-1}$. The probe beam is focalized on the input face of the crystal at the location of the waveguide. (a) Theory. (b) Experiments.

its steady-state regime. In fact, as explained in section 3.1, the refractive index contrasts $\Delta n_{x}$ and $\Delta n_{y}$ are already well developed at early times (see curve for $\tau=0.2$ on Fig. 3(c)). Later in time, the main effect is a global increase of the index while the moderate change of $\Delta n_{x}$ and $\Delta n_{y}$ influences very little the guiding properties. Fig. 8(a) has been obtained for three light stripes possessing the same peak intensities of $5 \mathrm{~mW} \mathrm{~cm}^{-2}$ as in Fig. 6(f). Since the refractive index contrast is higher along $x$, the probe beam is first focused in this dimension and a longer time is required to also guide the beam in the second transverse $y$-dimension. Nevertheless an efficient guiding is obtained for both dimensions at the final state.

The theoretical expectations are compared to experimental demonstrations in Fig. 8(b) for $E_{0}=3 \mathrm{kV} \mathrm{cm}^{-1}$. The experimental setup follows the architecture of Fig. 1 and more details can be found in Ref. [7]. The SBN crystal dimensions are $a \times b \times c=10 \times 5 \times 5 \mathrm{~mm}^{3}$. Thin $\mathrm{Cr}$-Au transparent electrodes are deposed in order to permit to shine light through them and to apply the electric field. The two control beams, at the wavelength $\lambda=532 \mathrm{~nm}$, are represented on Fig. 1 without considering the effects of diffraction or absorption. To avoid these practical limitations the waveguides are realized close to the crystal surfaces. The control beam 1 is the light structured by a Spatial (a)

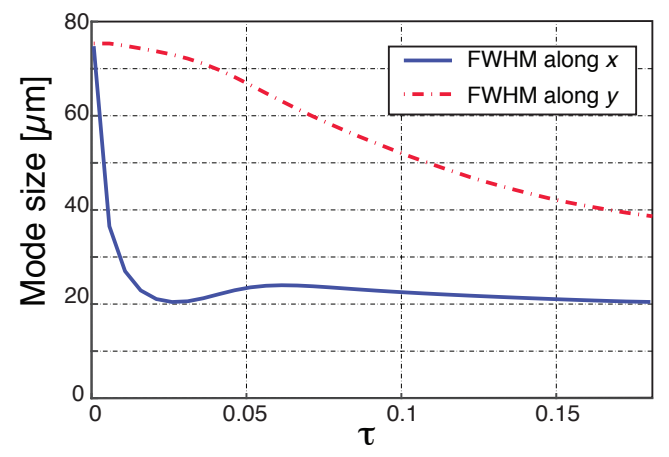

(b)

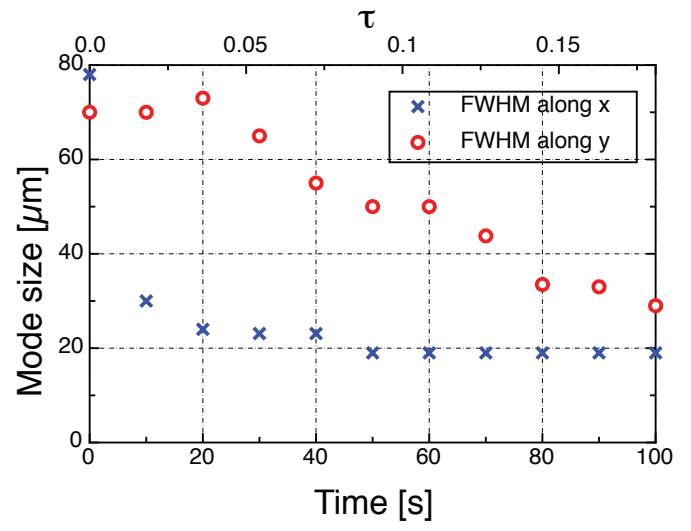

Figure 9: Time evolution of the probe beam size as for Fig. 8 but with $I_{2}=$ $0.5 I_{1}=2.5 \mathrm{~mW} \cdot \mathrm{cm}^{-2}$. (a) Theory. (b) Experiments.

Light Modulator and imaged by a cylindrical lens onto a plane inside the crystal. The imaged light stripe has a width of $25 \mu \mathrm{m}$ along the $c$-axis and is aligned parallel to the $z$-axis. The control beam 2 is composed by a dark region (produced by a thin wire) and is also imaged on the crystal to get a $25 \mu \mathrm{m}$ dark line. It propagates in direction nearly parallel to the crystal $c$-axis and is partially transmitted through the transparent electrodes. The intensities of the control beams can be separately adjusted to get similar illuminations as in the section 3.3 or to get other ratios between $I_{2}$ and $I_{1}$. A white incoherent light is used as the background illumination. The output face of the crystal is imaged on a CCD camera. Its images permit to determine the probe beam transverse profile in $x$ and $y$ directions after propagation in the $2 \mathrm{D}$ photo-induced waveguides. The experimental parameters (SBN properties and dimensions, light shapes and intensities, applied voltage) are therefore in accordance with the ones used in the simulations. Especially, the super-gaussian shape of the control beams in the simulations has been chosen to closely approximate the shape of the experimental light stripes.

Fig. 8(b) shows a good qualitative agreement with the theoretical predictions and with earlier experimental observations [7] and confirm the strong anisotropy in the dynamic of the guiding properties. Note that the upper axis in Fig. 8(b) allows direct comparison with the normalized time $\tau=t / T_{d}$. The dielectric response time $T_{d}$ of our weakly Ce-doped SBN 
(a)

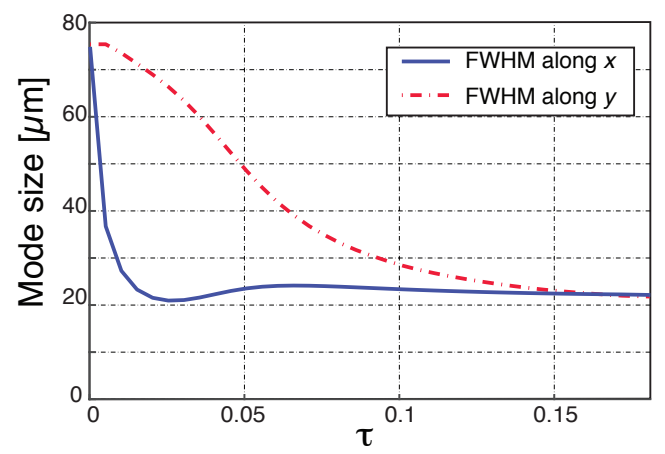

(b)

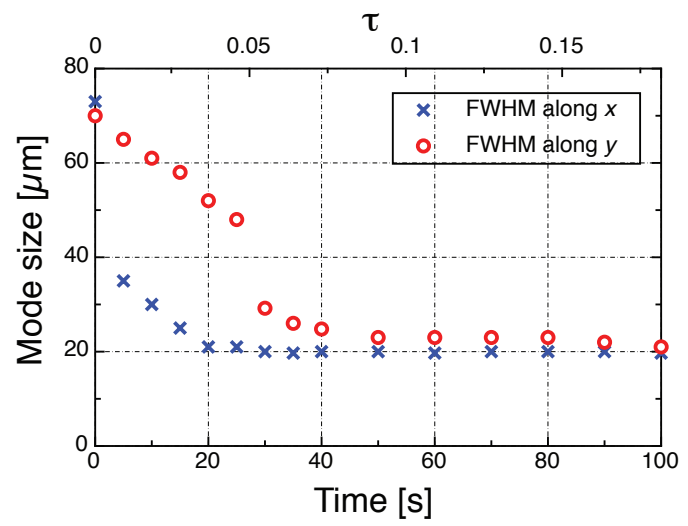

Figure 10: Time evolution of the probe beam size as for Fig. 8 but with $I_{2}=$ $2 I_{1}=10 \mathrm{~mW} \mathrm{~cm}^{-2}$. (a) Theory. (b) Experiments.

crystal was estimated by observing the dynamics of erasure of photorefractive holographic gratings recorded at small interfering angles in the same crystal and for $x$-oriented charge transport [21]. It was found that $T_{d} \approx 4400 / I_{\text {tot }}$ in the range of intensities used for our waveguide experiments. Here $T_{d}$ is in seconds and $I_{t o t}$ is the total intensity expressed in $\mathrm{mW} / \mathrm{cm}^{2}$.

It should be noted that anisotropic dynamics of self-focusing of spatial solitons has also been reported for a circular symmetric 2D illumination [26]. In our case, in contrast to soliton experiments, the intensities of the control beams 1 and 2 can be adjusted separately, which gives an additional flexibility to control this anisotropic dynamic. Other experiments with various contrast ratio $I_{2} / I_{1}$ have been tested and two examples are shown on Fig. 9 with $I_{2}=0.5 I_{1}=2.5 \mathrm{~mW} \mathrm{~cm}^{-2}$ and on Fig. 10 with $I_{2}=2 I_{1}=10 \mathrm{~mW} \mathrm{~cm}^{-2}$, all the others parameters being unchanged. Both simulations and experiments shows that for $I_{2}<I_{1}$ the confinement of the probe beam is smaller in $y$, confirming that $C$ is too weak in this case for an efficient channel waveguide. A slightly better confinement with $I_{2}=2 I_{1}$ than with $I_{2}=I_{1}$ is observed. Moreover, as expected theoretically, the formation time in $y$-direction is reduced when increasing $I_{2}$. We note also that our simulations indicate that an increase of the ratio $I_{2} / I_{1}$ permits to increase the index contrast ratio $C$ if this is necessary. However, for a finite transverse mobility this increase is weaker than expected by the simplified model of Ref. [7] which predicts $C \approx 1$ for $I_{2} \gg I_{1}$ and $\mu_{y}=0$.

The influence of the applied electric field has also been studied. Both simulations and experiments show that a stronger voltage than the $3 \mathrm{kV} \mathrm{cm}^{-1}$ used throughout this paper tends to accelerate the temporal dynamic in both waveguide dimensions, but there is a low impact on the mode size of the waveguide at the final stage. Calculations also show that a modification of the electric field around the value of $3 \mathrm{kV} \mathrm{cm}^{-1}$ has no influence on $C$ since both $\Delta n_{x}$ and $\Delta n_{y}$ are modified in the same proportion.

\section{Conclusion}

A numerical 3D model of the PR effect has been successfully used to model the formation dynamics of waveguides induced by lateral illumination of a biased PR crystal, both for planar and channel waveguides. The theoretical time-resolved study of a 1D-type illumination has shown an interesting transient dynamics. The formation of $1 \mathrm{D}$ waveguides reveals the emergence of a lower index region on each sides of the waveguide, similar to the one characteristic of a $2 \mathrm{D}$ illumination of a PR crystal. This leads to a faster confinement of the probe beam. This minima disappear in the steady-state regime as expected by standard $1 \mathrm{D}$ models, but the waveguide guiding properties remain almost unchanged during this evolution.

For the realization of $2 \mathrm{D}$ waveguides by lateral illumination, a complex structured illumination of the crystal is required, therefore a complete model was essential to reproduce the inducing rich build-up dynamics. Several parameters, such as a background intensity, the anisotropy of the conductivity, or the intensity ratio of the crossed control beams, were shown to influence the anisotropic dynamics as well as the final steadystate refractive index distribution. This improved understanding allows a better adjustment of the experimental parameters for generating efficient channel waveguides with similar confinement in both transverses dimensions. We have to stress that the transverse charge mobility (perpendicular to the direction of the applied electric field) not only influences the build-up process, but also limits the steady-state refractive index contrast of the waveguide in the transverse direction. This could offer a way to evaluate the mobility tensor by observing the steady state field distribution under different conditions.

[1] M. Morin, G. C. Duree, G. J. Salamo, M. Segev, Waveguides formed by quasi-steady-state photorefractive spatial solitons, Opt. Lett. 20 (1995) 2066-2068.

[2] M. Shih, Z. Chen, M. Mitchell, M. Segev, H. Lee, R. S. Feigelson, J. P. Wilde, Waveguides induced by photorefractive screening solitons, J. Opt. Soc. Am. B 11 (1997) 3091.

[3] E. Fazio, S. T. Popescu, A. Petris, F. Devaux, M. Ragazzi, M. Chauvet, V. I. Vlad, Use of quasi-local photorefractive response to generated superficial self-written waveguides in lithium niobate, Opt. Express 21 (2013) 25834-25840.

[4] P. Dittrich, G. Montemezzani, P. Bernasconi, P. Günter, Fast, reconfigurable light-induced waveguides, Opt. lett. 24 (1999) 1508-1510.

[5] P. Zhang, J. Zhao, D. Yang, D. Y. B. Li, X. Feng, Optical masks prepared by using a liquid-crystal light valve for light-induced photorefractive waveguides, Appl. Opt. 42 (2003) 4208-4211.

[6] G. Montemezzani, M. Gorram, N. Fressengeas, F. Juvalta, M. Jazbinsek, P. Günter, Light deflection and modulation through dynamic evolution of photoinduced waveguides, Opt. Express 16 (2008) 16646-16658. 
[7] M. Gorram, V. Coda, P. Thévenin, G. Montemezzani, Bulk channel-type reconfigurable light-induced waveguides recorded by crossed lateral illumination, Appl. Phys. B 95 (2009) 565-572.

[8] C. Ciret, M. Alonzo, V. Coda, A. A. Rangelov, G. Montemezzani, Analog to electromagnetically induced transparency and Autler-Townes effect demonstrated with photoinduced coupled waveguides, Phys. Rev. A 88 (2013) 013840.

[9] C. Ciret, V. Coda, A. A. Rangelov, D. N. Neshev, G. Montemezzani, Planar achromatic multiple beam splitter by adiabatic light transfer, Opt. Lett. 37 (2012) 3789-3791.

[10] M. Gorram, P. Thévenin, V. Coda, N. Fressengeas, G. Montemezzani, Reconfigurable one and two dimensional waveguides in strontium barium niobate induced by lateral illumination, Ferroelectrics 390 (2009) 10-17.

[11] G. Montemezzani, Optimization of photorefractive two-wave mixing by accounting for material anisotropies: $\mathrm{KNbO}_{3}$ and $\mathrm{BaTiO}_{3}$, Phys. Rev. A 62 (2000) 053803-053815.

[12] N. Fressengeas, J. Maufoy, G. Kugel, Temporal behavior of bidimensional photorefractive bright spatial solitons, Phys. Rev. E 54 (1996) 6866-6875.

[13] S. Lee, H. R. Yang, E. J. Kim, Y. L. Lee, C. H. Kwak, Kinetics of two wave mixing gain for moving grating technique in photorefractive $\mathrm{BaTiO}_{3}$ crystal, Opt. Express 16 (24) (2008) 19615-19628.

[14] F. Kalkum, K. Peithmann, K. Buse, Dynamics of holographic recording with focused beams in iron-doped lithium niobate crystals, Opt. Express 17 (2009) 1321-1329.

[15] M. Kösters, B. Sturman, D. Haertle, K. Buse, Kinetics of photorefractive recording for circular light beams, Opt. Lett. 34 (2009) 1036-1038.

[16] F. Devaux, V. Coda, M. Chauvet, R. Passier, New time-dependent photorefractive three-dimensional model: application to self-trapped beam with large bending, J. Opt. Soc. Am. B 25 (2008) 1081-1086.

[17] F. Devaux, M. Chauvet, Three-dimensional numerical model of the dynamics of photorefractive beam self-focusing in InP:Fe, Phys. Rev. A 79 (2009) 033823.

[18] F. Devaux, J. Safioui, M. Chauvet, Two-photoactive-center model applied to photorefractive self-focusing in biased $\mathrm{LiNbO}_{3}$, Phys. Rev. A 81 (2010) 013825 .

[19] N. V. Kukhtarev, V. B. Markov, S. G. Odulov, M. S. Soskin, V. L. Vinetskii, Holographic storage in electrooptic crystals. i. steady state, Ferroelectrics 22 (1979) 949-960.

[20] P. Yeh, Introduction to photorefractive nonlinear optics, Wiley, New York, 1993

[21] I. Biaggio, Recording speed and determination of basic materials properties, in: P. Günter, J.-P. Huignard (Eds.), Photorefractive Materials and Their Applications 2, Vol. 114 of Springer Series in Optical Sciences, Springer New York, 2007, pp. 51-81.

[22] K. Buse, A. Gerwens, S. Wevering, E. Krätzig, Charge-transport parameters of photorefractive strontium-barium niobate crystals doped with cerium, J. Opt. Soc. Am. B 15 (1998) 1674-1677.

[23] N. Korneev, D. Mayorga, S. Stepanov, A. Gerwens, K. Buse, E. Krätzig, Characterization of photorefractive strontium-barium niobate with nonsteady-state holographic photocurrents, Opt. Commun. 146 (1998) 215 219.

[24] A. A. Zozulya, D. Z. Anderson, Propagation of an optical beam in a photorefractive medium in the presence of a photogalvanic nonlinearity or an externally applied electric field, Phys. Rev. A 51 (1995) 1520-1531.

[25] F. S. Chen, Optically induced change of refractive indices in $\mathrm{LiNbO}_{3}, \mathrm{~J}$. Appl. Phys. 40 (1969) 3389-3396.

[26] E. Fazio, F. Renzi, R. Rinaldi, M. Bertolotti, M. Chauvet, W. Ramadan, A. Petris, V. I. Vlad, Screening-photovoltaic bright solitons in lithium niobate and associated single-mode waveguides, Appl. Phys. Lett. 85 (2004) $2193-2195$. 